\title{
Surgical palliation for endomyocardial fibrosis: early
} results

\author{
MS VALIATHAN, R SANKARKUMAR, KG BALAKRISHNAN, MP MOHANSINGH \\ From the Department of Cardiac Surgery and Cardiology, Sree Chitra Tirunal Institute for Medical Sciences \\ and Technology, Trivandrum, India
}

ABSTRACT Endomyocardial fibrosis is a fatal disease of obscure aetiology which is notably prevalent in Uganda, Brazil, Kerala State in India, and certain other parts of the world. The main disability in the disease results from fibrotic obliteration of ventricular chambers and insufficiency of the mitral and tricuspid valves. The poor response to medical treatment and the bleak prognosis of the patients led to the introduction of endocardiectomy and replacement of the atrioventricular valves as a palliative procedure by Dubost and colleagues. The present report on the early results in 17 patients from Kerala State would support the continued use of this palliative procedure in endomyocardial fibrosis with severe disability.

Endomyocardial fibrosis is a fatal disease which figures prominently among the cardiac causes of mortality and morbidity in several parts of the world, including the State of Kerala in India. While the aetiology, pathogenesis and geographical factors in incidence are poorly understood, its characterisation on the basis of clinical features, echocardiography, haemodynamic investigations, and histopathological and ultrastructural studies has made considerable progress in recent years.

The central problem in endomyocardial fibrosis is the progressive obliteration of the ventricular chambers by growing fibrous tissue, which entraps and immobilises the atrioventricular valves. The progressive disability of patients arises primarily from the insufficiency of the tricuspid or mitral valves, which may be affected singly or in combination. The poor response of this condition to medical treatment and its bleak prognosis led to the development of palliative surgery originally by Dubost and colleagues. ${ }^{1}$

In this paper we report our early results after palliative surgery for endomyocardial fibrosis in Kerala, which has a high prevalence of this disease.

Address for reprint requests: Professor MS Valiathan, Sree Chitra Tirunal Institute for Medical Sciences and Technology, Trivandrum-695011, India.

Accepted 3 February 1983

\section{Patients and methods}

Seventeen patients who were referred from the endomyocardial fibrosis registry of the institute for severe disability and refractory cardiac failure constituted this surgical series (table 1). All but one belonged to the non-paying group of patients at the institute on the basis of their low family income (below Rs 300 a month).

All had severe symptoms and belonged to functional class IV of the New York Heart Association (NYHA) classification. While eight patients had a history of vague febrile illness, none gave a clear history of rheumatic fever or polyarthritis. The dominant clinical features were dyspnoea, peripheral oedema, considerable cardiomegaly, and cachexia (table 2). One patient (case 11) showed massive splenomegaly, but without evidence of gastro-oesophageal varices or hypersplenism.

Electrocardiography ...Eight patients were in sinus rhythm and the others had atrial fibrillation. Three showed low-voltage complexes and all these had massive pericardial effusions. When the right ventricle or both ventricles were affected, right atrial enlargement was always appreciable. Left atrial enlargement was noted in patients whose left ventricle was affected. Ventricular hypertrophy was not noted in any of the patients.

Echocardiography In right ventricular disease visualisation of the tricuspid valve was usually difficult, 
Table 1 Profile of the patients

\begin{tabular}{llll}
\hline Case No & Sex & Age $(y)$ & $\begin{array}{l}\text { Form of endo- } \\
\text { myocardial fibrosis }\end{array}$ \\
\hline 1 & F & 38 & RV \\
2 & F & 38 & RV \\
3 & M & 13 & RV \\
4 & M & 16 & RV \\
5 & M & 12 & RV \\
6 & G & 17 & BV \\
7 & F & 24 & BV \\
8 & F & 32 & LV \\
9 & M & 35 & LV \\
10 & M & 38 & RV \\
11 & F & 55 & RV \\
12 & F & 23 & RV \\
13 & M & 25 & LV (early RV) \\
14 & M & 28 & RV \\
15 & M & 7 & LV (early RV) \\
16 & F & 23 & LV \\
17 & & & \\
\hline
\end{tabular}

$R V$-right ventricular; $L V$-left ventricular; $B V$-biventricular.

Table 2 Clinical features

\begin{tabular}{ll}
\hline Clinical features & No of patients \\
\hline Dyspnoea & 17 \\
Cardiothoracic ratio $>60 \%$ & 16 \\
Peripheral oedema & 10 \\
Ascites & 8 \\
Cachexia & 7 \\
Pulmonary oedema & 4 \\
Chest pain & 1 \\
Thromboembolism & 0 \\
\hline
\end{tabular}

owing to the displacement or destruction (or both) of the valve apparatus by the disease process. Ventricular septal motion was paradoxical in right ventricular disease, indicating tricuspid regurgitation. Steep posterior motion of the left ventricular posterior wall in early diastole was a common feature in left ventricular disease.

Cardiac catheterisation A notable feature was the loss of ventricular compliance with consequent

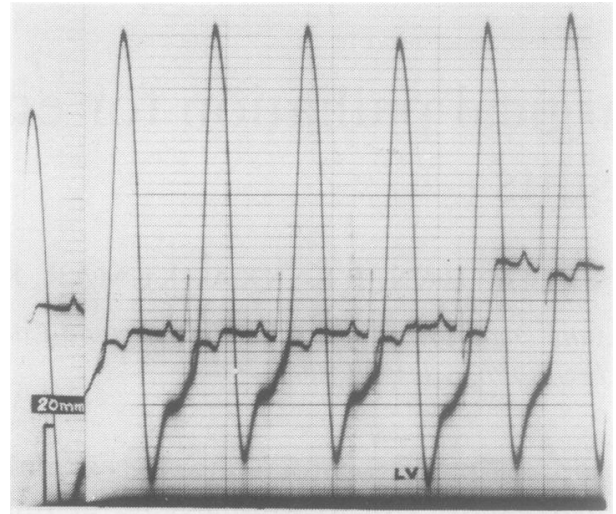

Fig 2 Left ventricular pressure trace.

increase of the filling pressures. Initially the enddiastolic pressure alone was raised, but in the later $ᄃ$ stages the early diastolic pressure was also increased. With further progression of the disease, especially in the right ventricle, systolic function was affected, resulting in atrialisation of the ventricular pressure curve (fig 1). A similar degree of loss of systolic function is not compatible with life in left ventricular disease, and the left ventricular pressure curve showed diastolic elevation only (fig 2 ). None of the patients showed associated cardiovascular anomalies.

Angiocardiography Right ventricular disease was characterised by obliteration of the right ventricular inflow chamber and enormous enlargement of the right atrium, which opened directiy into the dilated outflow of the right ventricle (fig 3 ). Disease of the left ventricle caused irregular filling defects and obliteration of the apical segment, with varying degrees of mitral insufficiency (fig 4). One patient

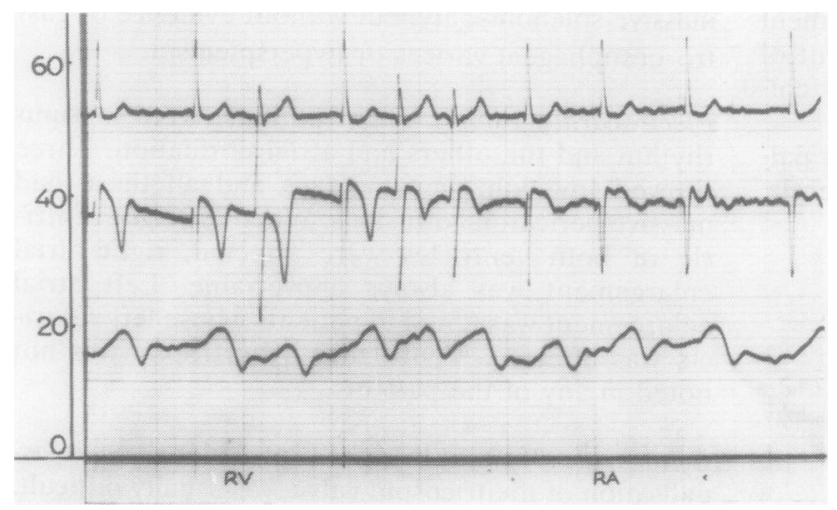

Fig 1 Lower trace: withdrawal pressure tracing from pulmonary artery to right atrium $(\mathrm{mm} \mathrm{Hg})$; middle trace: intracardiac electrocardiogram. 


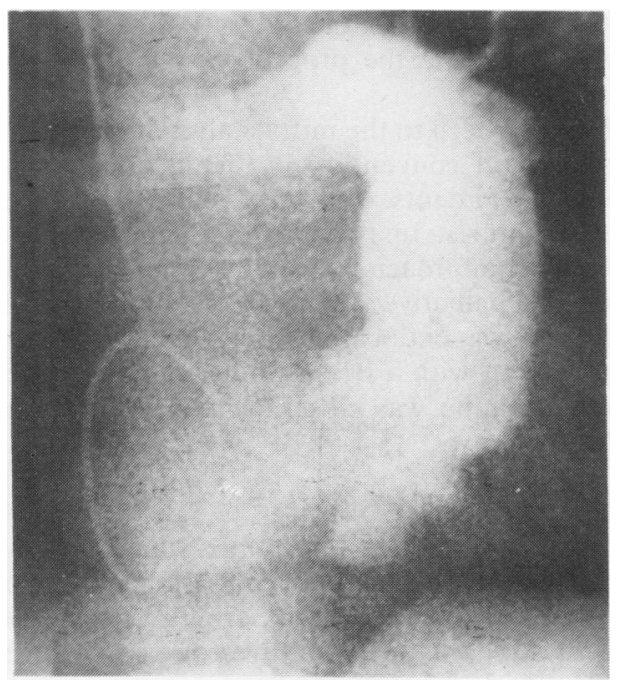

Fig 3 Angiocardiogram of a patient with right ventricular endomyocardial fibrosis.

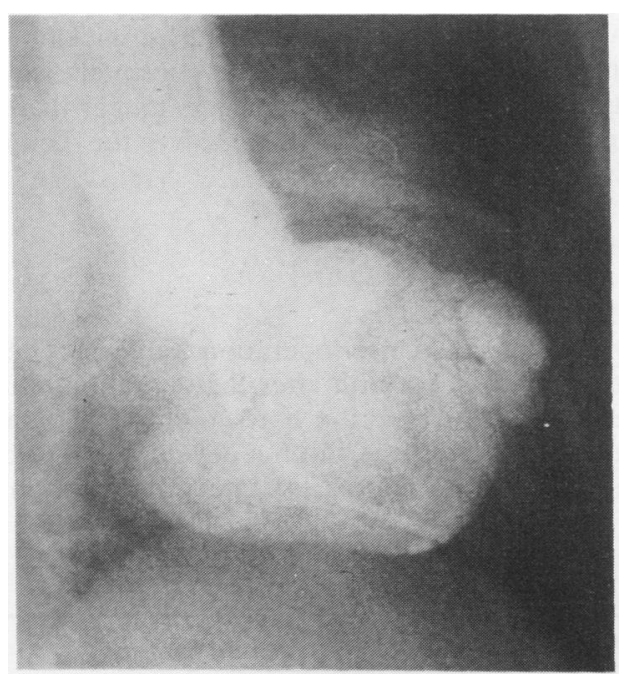

Fig 4 Angiocardiogram of a patient with left ventricular endomyocardial fibrosis.

with left ventricular endomyocardial fibrosis had changes suggestive of a pseudoaneurysm of the subannular segment of the posterior wall of the left ventricle. The outpouched segment showed no paradoxical motion but was calcified.

\section{Surgical treatment}

At the time of referral for operation, all patients in the series had been having vigorous decongestive treatment for several years. Anaesthetic induction was with sodium pentothal and maintenance with nitrous oxide and oxygen mixture, morphine, and pancuronium bromide. Standard cardiopulmonary bypass was used with a low-blood prime, disposable bubble oxygenator, and systemic hypothermia of $28^{\circ} \mathrm{C}$. Multidose potassium cold cardioplegia was routinely used. The pump time and aortic occlusion time averaged two hours and one hour respectively, the longest being $3^{1 / 2}$ and $2^{1 / 2}$ hours (case 7).

\section{SURGICAL FINDINGS}

In right ventricular endomyocardial fibrosis the right atrium was massively enlarged and thin walled (fig $5)$. The right ventricle often showed a spoon-shaped depression over the inflow region with dilatation of the outflow tract and pulmonary artery. The interior of the right atrium frequently contained clots or organised thrombi, which showed pedunculation in one patient (No 5). The posterior, septal, and anterior leaflets and chordae of the tricuspid valve, in that order of frequency and intensity, were bound to the ventricular endocardium, which was lined uniformly or in patches by white, leathery fibrous tissue. Not infrequently the endocardial tissue showed calcified areas. The valve leaflets and chordae when unattached were thin. The papillary muscles were invariably incorporated in the fibrotic reaction, which seemed to affect the posterior wall and apex preferentially, even though no part of the endocardium was immune from the process. The right ventricular outflow tract was dilated and largely free from fibrosis of the endocardium. In two patients (Nos 13 and 15) with dominant left ventricular endomyocardial fibrosis the right ventricle showed dilatation, mild tricuspid regurgitation, and abnormalities in

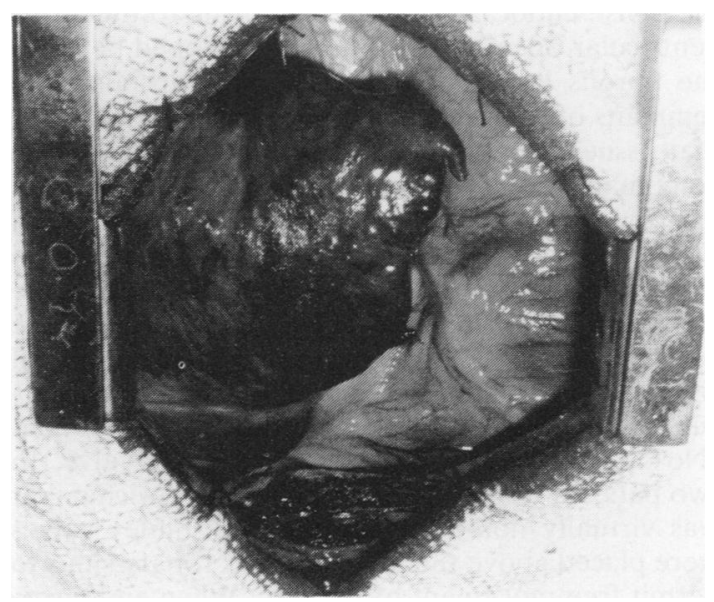

Fig 5 Operative appearance of greatly enlarged right atrium. 
contractility during angiocardiography. The right ventricle of these patients showed a dilated tricuspid annulus; a normal tricuspid valvular apparatus; and two small, thin areas of fibrosis on the side and base of the anterior papillary muscle. These areas were removed without damage to the integrity of the papillary muscle and showed histopathological changes consistent with endomyocardial fibrosis.

Left ventricular endomyocardial fibrosis caused similar changes, though the obliteration of the chamber was always less severe than in the right ventricle. The fibrosis with patchy calcification was seen commonly to affect the apex, papillary muscles, and posterior wall. The posterior leaflet and chordae of the mitral valve were incorporated in the fibrosed endocardium. The anterior leaflet and chordae were usually thin, but the fibrotic change of the anterior papillary muscle tended to compromise its free mobility. In one patient (No 7) the anterior mitral leaflet also showed severe calcification with appreciable fusion and shortening of its chordal mechanism, in addition to the classic findings of endocardial fibrosis. Another showed a subannular outpouching of the posterior ventricular wall with severe endocardial fibrosis of the left ventricular chamber (case 17).

\section{OPERATION}

In cases of right ventricular endomyocardial fibrosis bicaval and aortic cannulation was often difficult owing to the enormous enlargement, intracavitary thrombi, and thin wall of the right atrium. When the right atrium had been opened thrombi were carefully removed and the unattached sections of the tricuspid leaflets incised near the annulus. If the leaflet was completely fused, an incision was made in the dense endocardial tissue just distal to the atrioventricular opening and a plane developed between the fibrous layer and subjacent myocardium. The remnants of tricuspid leaflets and the thick endocardial tissue were dissected off the myocardium by a combination of sharp and blunt manoeuvres. The excision of the fibrous tissue was limited to the distal half of the ventricular septum to avoid damage to the conduction system, which had occurred after the complete excision of septal endocardium in one patient (No 1). After removal of the endocardial tissue a Björk-Shiley valve was inserted (with interrupted mattress sutures) in all except one patient (No 5), who received an Ionescu-Shiley valve. In two patients whose right ventricular inflow chamber was virtually non-existent the Björk-Shiley valves were placed above the level of the coronary sinus to permit free movement of the disc. When endomyocardial fibrosis affected both the left and the right ventricles the left ventricle and mitral valve were approached through the interatrial septum as the first step, before the procedure was performed on the right side.

The approach to the mitral valve and left ventricle was effected conventionally behind the interatrial groove in patients with left ventricular endomyo- $\mathbb{D}$ cardial fibrosis. In two patients with biventricular disease the approach was through the interatrial sep- os tum. The palliative procedure in the left ventricle consisted of endocardiectomy and mitral valve replacement with a Björk-Shiley valve and the surgical technique was closely similar to that for the right side.

\section{POSTOPERATIVE COURSES}

All patients required inotropic support with iso- $\vec{N}$ prenaline or dobutamine and assisted ventilation 0

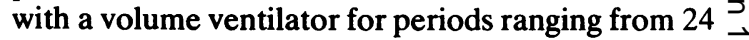
to 72 hours. The postoperative course was generally $\subset$ stormy and weaning from supportive measures difficult. Five patients developed low cardiac output insidiously during the second postoperative week owing to massive haemorrhagic pericardial effusions, which were drained surgically with immediate improvement. The hospital stay of patients averaged 20 days and was longer than that of patients receiving prosthetic valves for rheumatic valve disease (10-12 days).

\section{Results}

\section{OPERATIVE DEATHS}

Five patients died after operation (table 3 ). The first patient died one month after the operation. During the postoperative period her symptoms had shown gradual improvement, but the development of complete heart block had necessitated implantation of a

Table 3 Postoperative deaths

\begin{tabular}{cccl}
\hline Case No & Age $(y)$ Procedure & Cause of death \\
\hline 1 & 38 & $\begin{array}{l}\text { TVR, RV endo- } \\
\text { cardiectomy }\end{array}$ & $\begin{array}{l}\text { Total thrombosis of } \\
\text { Björk-Shiley valve } \\
\text { in tricuspid position; } \\
\text { AV dissociation; } \\
\text { died one month after } \\
\text { operation }\end{array}$ \\
4 & 14 & $\begin{array}{l}\text { TVR, RV endo- } \\
\text { cardiectomy } \\
\text { TVR, RV endo- }\end{array}$ & $\begin{array}{l}\text { Failed to come off } \\
\text { bypass } \\
\text { Bleeding: AV dissociation;0 } \\
\text { died 3 h after operation }\end{array}$ \\
10 & 12 & $\begin{array}{l}\text { cardiectomy } \\
\text { TVR, RV endo- } \\
\text { cardiectomy } \\
\text { with minimal support; } \\
\text { developed bizarre ventricular } \\
\text { arrhythmia and low output } \\
\text { on 4th postoperative day } \\
\text { Low output; died 12 h } \\
\text { after operation }\end{array}$ \\
\hline
\end{tabular}

$T V R \rightarrow$ tricuspid valve replacement; $R V \rightarrow$ right ventricular; $A V$-atrioventricular. 
pacemaker. Her death was sudden and at necropsy the Björk-Shiley valve was found to be totally thrombosed on both the atrial and the ventricular surface.

The second patient had massive ascites and pericardial effusion at the time of operation. After thrombectomy of the large right atrium, endocardiectomy of the right ventricle, and replacement of the tricuspid valve with a No 27 Björk-Shiley valve he failed to come off bypass despite the use of various supportive drugs. Necropsy showed no pathological features other than those of right ventricular endomyocardial fibrosis, with evidence of chronic venous congestion in the viscera.

Patient No 5 had massive ascites, right atrial enlargement, and obliteration of the right ventricular inflow chamber. After thrombectomy of the right atrium, endocardiectomy of the right ventricle, and tricuspid valve replacement with a No 27 Ionescu-Shiley valve, he developed atrioventricular dissociation and severe bleeding from the right atriotomy repair, which was controlled with difficulty. Even though he came off bypass with inotropic support, his cardiac output dropped sharply three hours later and he died. Necropsy showed one post of the Ionescu-Shiley valve to be intruding on the septum of a diminutive right ventricular chamber.

Patient No 10 had ascites, pericardial effusion, varicosities of the leg veins, and other advanced signs of congestive heart failure. At operation right ventricular endocardiectomy and tricuspid valve replacement were carried out with a No 27 BjörkShiley valve. His immediate recovery was satisfactory and he required minimal inotropic support. On the fourth day he developed low cardiac output and bizarre ventricular arrhythmia, which were unresponsive to treatment. Necropsy showed the valve to be well placed and there were no other important findings.

The youngest patient (No 16) had massive enlargement of the right atrium, tricuspid insufficiency, and ascites. After thrombectomy of right atrium, right ventricular endocardiectomy, and tricuspid valve replacement he came off bypass with inotropic support. In the immediate postoperative period he had several episodes of atrial fibrillation with rapid ventricular response and progressive drop in cardiac output. Death occurred 12 hours later. Necropsy showed the prosthetic valve to be well placed, with early changes of endomyocardial fibrosis in the left ventricular apex.

\section{LATE DEATH}

One patient (No 7), whose calcific mitral valve and endocardial lesions suggested a combination of rheumatic valvulitis and endomyocardial fibrosis, remained well until four months after operation, when he was readmitted with severe dyspnoea. He died within a few hours of admission with intractable pulmonary oedema and necropsy showed complete thrombosis of the Björk-Shiley valve. His prothrombin time on readmission was the same as the control value.

\section{SURVIVORS}

Twelve patients survived operation and were followed up regularly in the institute. Routine followup included clinical examination, two-dimensional echocardiography, and screening for evaluation of valve disc motion. All the survivors showed appreciable reduction in cardiomegaly, oedema, ascites, hepatomegaly, and other signs of cardiac failure (figs $6 a$ and $6 b$ ). Apart from the patient who died in the fourth month, the functional improvement was satisfactory and 11 survivors improved by at least one

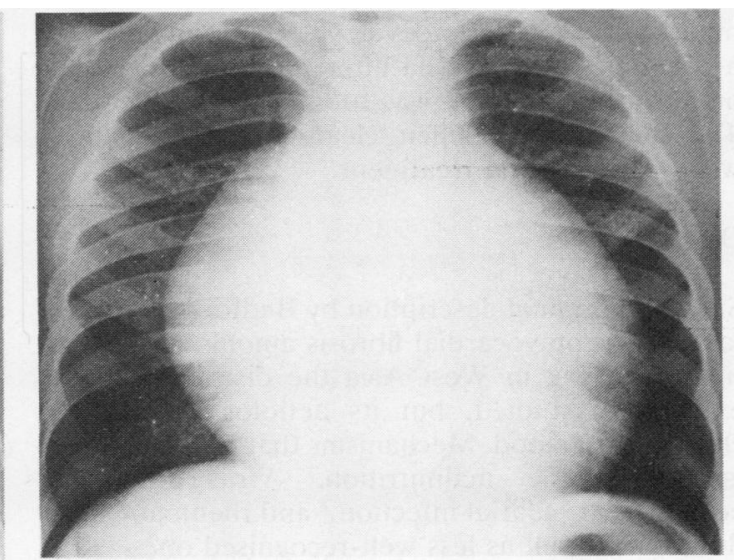

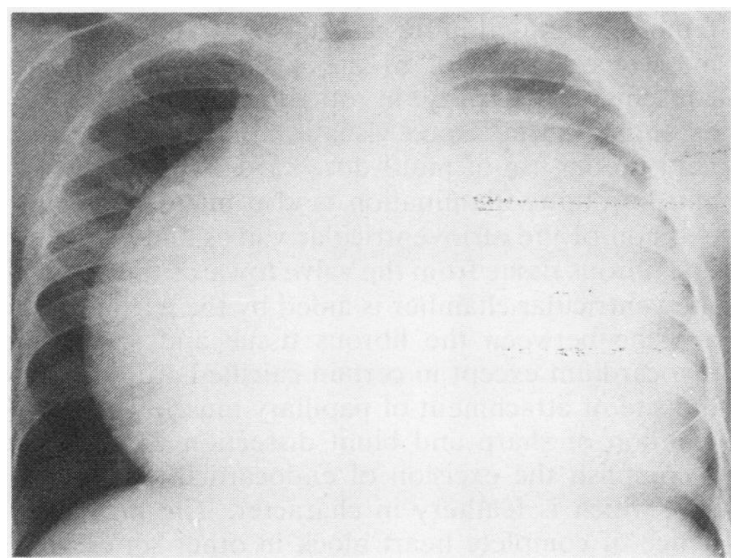

Fig 6 (a) Preoperative and (b) postoperative chest radiographs of patient No 6. 


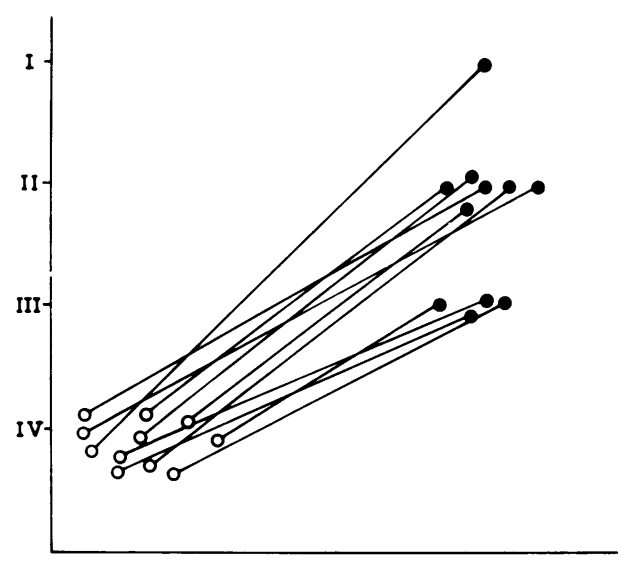

Fig 7 Preoperative (open circles) and postoperative (closed circles) functional state (NYHA classification) of surviving patients.

grade of the NYHA classification within three months of the operation. The postoperative recovery and ability to resume normal activities were dramatic in four patients. The functional state of the survivors three to 16 months after operation is indicated in figure 7. Decongestive treatment, which before operation had consisted of severe salt restriction, administration of multiple diuretics in large daily doses, and digitalis treatment, was greatly simplified after operation, patients receiving only 20-40 $\mathrm{mg}$ frusemide two or three times a week. Eight had returned to work as housewives or were at school or carrying on small trades at their last follow-up visit.

One patient (No 9) who had had mitral valve replacement developed a transient left hemiparesis two months after operation. This episode resolved gradually and resulted in little recognisable neurological disability. Patient No 6, who had had double valve replacement for biventricular endomyocardial fibrosis, developed a transient left hemiparesis and aphasia after three months, when his prothrombin time was found to be inadequate. The neurological deficit cleared completely in a week with medical treatment.

\section{Discussion}

Since the original description by Bedford and Kons$\operatorname{tam}^{2}$ of endomyocardial fibrosis among the African troops serving in West Asia the disease has been extensively studied, but its aetiology is not yet clearly understood. Mechanisms that have been suggested include malnutrition, ${ }^{3}$ virus infection, ${ }^{4}$ eosinophilia, ${ }^{5}$ filarial infection, ${ }^{6}$ and rheumatic heart disease, ${ }^{7}$ as well as less well-recognised ones.

In the classic study from Kampala, most patients with endomyocardial fibrosis were found to present $\stackrel{x}{x}$ soon after the onset of symptoms. ${ }^{8}$ In that series $\underset{\vec{\rho}}{\vec{\rho}}$ $44 \%$ died in less than one year and $40 \%$ from one to $\stackrel{\frac{9}{+}}{\circ}$ three years after the onset of symptoms. The endomyocardial fibrosis registry of this institute would $\frac{\bar{\sigma}}{\bar{\omega}}$ tend to confirm the uniformly poor prognosis of the $\frac{\vec{\nabla}}{\mathbb{D}}$ disease and its resistance to conventional treatment $\propto$ with decongestive measures. While our follow-up is necessarily short our preliminary experience cor- $\vec{\circ}$ roborates the encouraging results of Dubost and col- leagues ${ }^{1}$ with palliative surgery for this condition.

The high mortality rate for valve replacement and $\stackrel{\circ}{P}$ endocardiectomy in our series was confined to the $\vec{x}$ patients with right ventricular endomyocardial $\omega_{\infty}^{\omega}$ fibrosis, who had advanced cardiac failure with $\%$ obliteration of the right ventricular inflow region $\overrightarrow{\mathrm{N}}$ and a characteristic depression on the external sur- $\vec{O}$ face of the heart. This is probably because patients $\frac{}{\triangle}$ with the right ventricular form of the disease tend to present in the late stages, as right heart failure is tolerated better and for longer periods. By contrast $\widehat{\phi}$ in left ventricular endomyocardial fibrosis the pre- $\vec{\bullet}$ sentation is likely to be early as dyspnoea and $\underset{\omega}{\infty}$ orthopnoea are less well tolerated. The later results of Dubost and colleagues ${ }^{9}$ and our experience with both left ventricular and the earlier stages of right ventricular endomyocardial fibrosis would suggest that the operative mortality and morbidity can be $\stackrel{\varnothing}{\mathbb{Q}}$ reduced significantly by palliative surgery in patients $\varrho$ with right ventricular endomyocardial fibrosis $\overrightarrow{\overrightarrow{0}}$ before they reach the stage of advanced cardiac fail- $\exists$ ure. For optimum surgical results it is also necessary that patients are admitted several days before operation and are treated vigorously for the control of cardiac failure.

During operation the frequent presence of $\stackrel{\mathscr{N}}{x}$ thrombi in the large and tense right atrium will $\frac{3}{3}$ necessitate great care in the insertion of vena caval catheters. It may on occasion be prudent to use $\mathcal{O}$ femoral cannulation for arterial return owing to the massive enlargement of the right atrium and the $\frac{\text { 의 }}{8}$ consequent difficulty in obtaining access to the ascending aorta. Good visualisation in a quiet, dryㅡㅡㄹ field by the use of multi-dose cardioplegic solution $N$ and fibreoptic illumination is also mandatory. The excision of the atrioventricular valves and endocar- 0 dial fibrous tissue from the valve towards the apex of $\omega$ the ventricular chamber is aided by the existence of a plane between the fibrous tissue and subjacento myocardium except in certain calcified areas and at the site of attachment of papillary muscles. A com-? bination of sharp and blunt dissection will readily ${ }^{\circ}$ accomplish the excision of endocardial fibrous tissue, which is leathery in character. The high inci- $\stackrel{\mathbb{Q}}{\stackrel{\oplus}{\oplus}}$ dence of complete heart block in other series ${ }^{9}$ and $\Phi$ the occurrence of heart block in our first patient, 
who had complete excision of the fibrous endocardium, have prompted us to be conservative in the resection of fibrous tissue from the septal wall of the right ventricle. As an improved chamber size after radical endocardiectomy has yet to be clearly demonstrated, we have felt justified in limiting the excision of fibrous tissue in cases of right ventricular endomyocardial fibrosis to the distal half of the septal wall to avoid the serious complication of complete heart block.

In replacing the tricuspid or mitral valve it is necessary to choose a low-profile valve, as in our experience the chamber size is too small to accommodate any other design. We noted in fact that a supra-annular placement of the Björk-Shiley valve was advantageous and necessary in two patients whose right ventricular inflow region was largely obliterated and would not permit the free movement of the tilting disc. The use of a tissue valve was followed by the intrusion of the valve post on the septum, complete heart block, and mild incompetence of the valve in one patient (No 5) with severe right ventricular endomyocardial fibrosis, who died shortly after operation. If, however, the chamber size is adequate, as seen in the earlier stages of endomyocardial fibrosis, a tissue valve with tall posts may be suitable.

The postoperative course of these patients is marked by cardiac failure, arrhythmias, and respiratory problems. In contrast to the patients who undergo valve replacement for rheumatic valvulitis, patients with endomyocardial fibrosis need prolonged inotropoic and ventilatory support. Moreover, during the second postoperative week, when they are having oral anticoagulants, they have a tendency to develop large haemorrhagic pericardial effusions, which call for prompt surgical decompression.

Dubost and colleagues reported an operative mortality rate of $15 \%$ and an incidence of atrioventricular dissociation of $35 \%$ in their surgical series. Nevertheless, they concluded on the basis of a follow-up of nine years that the surgical procedure was preferable to continued medical treatment. The operative mortality rate in the present series was $30 \%$, all the deaths being confined to patients with right ventricular endomyocardial fibrosis-none of whom was denied surgery because of the advanced stage of the disease. Postoperative atrioventricular dissociation was limited to two patients, one of whom had complete excision of the septal endocardium and the other insertion of a tissue valve with tall commissural posts. The problem of complete heart block has not been encountered since we curtailed endocardial excision on the septum and avoided valves with a tall profile.

Postoperative thromboembolism in our series accounted for two deaths in patients with right ventricular endomyocardial fibrosis and two minor events in patients with left ventricular endomyocardial fibrosis and biventricular disease respectively. In three of these thromboembolisms the prothrombin time was not sufficiently prolonged or was grossly below the therapeutic range at the time of the event, owing to the irregular intake of oral anticoagulants. The rate of thromboembolism may therefore overestimate the thrombogenic potential of the prosthetic valve and the procedure of valve replacement in endomyocardial fibrosis.

While we cannot make predictions about the durability of clincal improvement or the recurrence of myocardial fibrosis on the basis of early observations, these do tend to suggest that surgical palliation has a useful role in the management of patients with endomyocardial fibrosis who are very ill and are unresponsive to medical treatment. The continuing improvement in operative results with greater surgical experience and the dramatic change in the quality of life of the survivors have encouraged our group to continue the policy of palliative surgery for this condition.

\section{References}

${ }^{1}$ Dubost C, Maurice P, Gerbaux A, et al. The surgical treatment of constrictive fibrous endocarditis. Ann Surg 1976;184:303-7.

${ }^{2}$ Bedford DE, Konstam GLS. Heart failure of unknown aetiology in Africans. Br Heart $J$ 1946;8:236-7.

${ }^{3}$ Shaper AG, Coles RM. The tribal distribution of endomyocardial fibrosis in Uganda. $\mathrm{Br}$ Heart $\mathrm{J}$ 1965;27:121-7.

${ }^{4}$ Williams AW, Ball JD, Davies JNP. Endomyocardial fibrosis in Africa: its diagnosis, distribution and nature. Trans $R$ Soc Trop Med Hyg 1954;48:290-311.

5 Vakil RJ. Cardiovascular involvement in tropical eosinophilia. Br Heart J 1961;23:578-86.

${ }^{6}$ Ive FA, Willis AJP, Ikeme AC, Brockington IF. Endomyocardial fibrosis and filariasis. $Q \mathrm{~J}$ Med 1967;36:495-516.

${ }^{7}$ Abrahams D, Brigden W. Syndrome of mitral incompetence, myocarditis, and pulmonary hypertension in Nigeria. Br Med J 1961;ii:134-9.

${ }^{8}$ D' Arbela PG, Mutazindwa T, Patel AK, Somers K. Survival after first presentation with endomyocardial fibrosis. Br Heart J 1972;34:403-7.

${ }^{9}$ Dubost C, Prigent C, Gerbaux A, et al. Surgical treatment of constrictive fibrous endocarditis. J Thorac Cardiovasc Surg 1981;82:585-91. 